\title{
A Storage Management for Mining Object Moving Patterns in Object Tracking Sensor Networks
}

\author{
Chih-Chieh Hung and Wen-Chih Peng* \\ Department of Computer Science \\ National Chiao Tung University \\ Hsinchu, Taiwan, ROC \\ E-mail:\{hungcc@csie.nctu.edu.tw,wcpeng@cs.nctu.edu.tw\}
}

\begin{abstract}
One promising application of sensor networks is object tracking. Because the movements of the tracked objects usually show repeating patterns, we propose a heterogeneous tracking model, referred to as HTM, to efficiently mine object moving patterns and track objects. To ensure the quality of moving patterns, we develop a storage management to facilitate mining object moving patterns. Specifically, we explore load-balance feature to store more moving data for mining moving patterns. Once a storage of a cluster head is occupied by moving data, we devise a replacement strategy to replace the less informative patterns. Simulation results show that HTM with storage management is able not only to increase the accuracy of predition but also to save more energy in tracking objects.
\end{abstract}

\section{Introduction}

Object tracking is one of the killer applications for wireless sensor networks. Various energy conservation schemes for object tracking sensor networks have been extensively studied in the literature [3][5]. In this paper, we concentrate at the prediction-based object tracking sensor network. The prediction-based object tracking sensor networks relies on certain prediction mechanisms to achieve energy saving. We argue that tracked objects such as human or animals tend to have their own moving patterns since the behaviors of human or animals are likely to be regular [4]. Thus, efficient techniques for obtaining moving patterns of objects are very important for energy conservation in object tracking sensor networks. Various data mining techniques have been explored in the literature [1]. However, these prior works mostly require data to be collected at one centralized server, leading to a significant amount of energy con-

\footnotetext{
*The corresponding author of this paper.
}

sumption in data collection. Our goal is to propose an efficient data mining mechanism for deriving object moving patterns in object tracking sensor network and utilize the object moving patterns for energy saving prediction-based object tracking sensor networks.

To facilitate collaborative data collection processing in object tracking sensor networks, cluster architectures are usually used to organize sensor nodes into clusters (with each cluster consisting of a cluster head and sensors). Similar to [2], we consider the sensor network which consists of heterogeneous nodes of various functions and roles and thus propose a heterogeneous tracking model (referred to as HTM) in the cluster architecture, in which a large number of inexpensive sensor nodes perform sensing operations and a limited number of heterogeneous nodes (standing for cluster heads) offer data collection and mining capabilities. For scalability, the cluster heads recursively form a hierarchical architecture for efficiently mining and queries. As such, the higher-level cluster heads will maintain coarse object moving patterns and the low-level cluster heads will have more precise object moving patterns. Based on the obtained object moving patterns, the cluster heads predict the object movements. If the prediction fails, a recovery procedure will be executed by waking up sensor nodes within the coverage region of the cluster head. Based on HTM, only a bound number of sensor nodes need to participate in the recovery procedure.

In HTM, cluster heads should keep as many moving log data as possible in their clusters. Note that cluster heads also have storage constraint and the amount of data will affect the effect of pattern mining. Notice that the conventional hierarchy clustering architectures (i.e., the level $i$ cluster head is chosen among these level ( $i-1)$ cluster heads) suffer from the storage load unbalance problem. Assume that the storage space of a cluster head is $S$ and the height of hierarchy is $k$, there is always a cluster head (called heavy node) which stores information of all level in the hierarchy 
and thus can only use $\frac{S}{k}$ to store information of each level. The heavy node can only mine patterns with shorter term than these cluster heads that only store information of one or fewer levels. In this paper, we develop a storage management, which consists of two modules: load-balance storage scheme and one replacement strategy. In the load-balance scheme, we address how to mine and maintain the informative patterns under the storage constraints of cluster heads. We develop a replace strategy to replace less informative patterns when the storage space of a cluster head is full.

The rest of the paper is organized as follows. Preliminary is described in Section 2. Storage management for HTM is presented in Section 3. Performance study is conducted in Section 4. This paper concludes with Section 5.

\section{Preliminaries}

In this section, we briefly illustrate the tracking mechanism used in HTM. Assume that low-end sensor nodes and cluster heads have unique sensor identifications and these sensor nodes are well time-synchronized. Suppose that each low-end sensor node is a logical representation of a set of sensor nodes which collaboratively detect an object. When a low-end sensor detects an object, this sensor node will inform the corresponding level-0 cluster heads of the detected object identification, object arrival time and its sensor identification. In other words, the location of an object is represented as a sensor identification and the moving log for each object is viewed as a moving stream, which is composed of a series of symbols. Since the cluster heads still have the storage constraint, in this paper, we adopt the variable memory Markov (referred to as VMM) model, which has been shown to be very effective in capturing dependences and in obtaining sequential models in one scan, to discover object moving behavior in every cluster head.

Due to the storage constraint in cluster head and the dependence of movements, mining object moving patterns can be regarded as a VMM model training. For each object, we use a variation of a suffix tree called emission tree [6] to maintain its VMM model and one corresponding buffer in a cluster head is used to hold the most recently segment of moving records. Each edge of an emission tree represents a moving record (i.e., sensor id) appearing in the moving path. A tree node of an emission tree is denoted as a concatenation of the edge labels from the node to the root. In other words, a tree node labeled as $r_{k} \ldots r_{2} r_{1}$ can be reached from the traversal path from root $\rightarrow r_{1} \rightarrow r_{2} \rightarrow \ldots \rightarrow r_{k}$. Each tree node will maintains the occurrence number of its label in the moving path. Furthermore, each tree node also records the conditional probabilities of all consecutive moving records given the node label as the preceding segment. For example, according to the conditional probabilities of consecutive moving records of node EF in Figure 1, it can

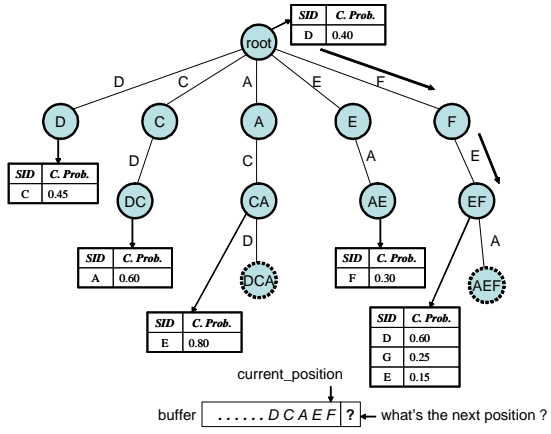

Figure 1. The resulting emission tree. The nodes with dash circle are immature. Aother nodes are mature.

be verified that $\mathrm{P}(\mathrm{D} \mid \mathrm{EF})$ is 0.60 . Consequently, if the most recently moving record is $\mathrm{EF}$, one can estimate the consecutive movement to be $\mathrm{D}$.

VMM model is trained on the fly and not all the tree nodes are suitable for predicting. There are two kind of nodes in an emission tree: mature node and immature node. Mature nodes are those tree nodes whose the conditional probabilities are stable. When predicting the next movement, only mature node is participated in prediction. Following the above example, the mature node EF is used to predict instead of the immature node AEF. To justify whether a node is mature or not, we explore $L_{\infty}$ distance, which is defined as follows:

Definition 1: For a node y with the corresponding probability table denoted as $x$ and the number of tuples in table $\mathrm{x}$ is $\mathrm{n}, L_{\infty}\left(x, x^{\prime}\right)=\max _{i=1, \ldots, n}\left(\left|d_{i}^{x}-d_{i}^{x^{\prime}}\right|\right)$, where $\mathrm{d}_{i}^{x}$ represents the probability value for tuple $i$ in table $x$ and table $x^{\prime}$ is the probability table after updating.

Definition 2: For node $y$ and given two application dependent parameters $\alpha$ and $\beta$, if $L_{\infty}\left(x, x^{\prime}\right) \leq \alpha$ for $\beta$ times of successive updates, then node $y$ is a mature node.

\section{Storage Management for HTM}

Since the quality of moving patterns are the dominant factor for HTM, the storage management is thus an important issue for HTM. In Section 3.1, we develop a new storage scheme for HTM. In Section 3.2, we devise a replacement strategy to keep the most informative patterns when memory space of a cluster head is full.

\subsection{Load-Balance Data Storage Scheme}

To facilitate to understand the concept, we assume that cluster heads are grid deployed and the degree of hierar- 
chy is four ${ }^{1}$. To avoid the heavy node problem of conventional hierarchy architectures, we must consider the number of levels of information a cluster head stores. Once a cluster head stores too much information, we tend to separate data flows to other cluster heads stored fewer information. Based on this concept, we propose a storage load-balanced scheme for HTM:

Given child cluster heads $C_{i}$ in level $i$ and the coordinate of sink, the parent cluster head of $C_{i}$ is assigned by the following rules: If there exist a cluster head $v \in C_{i}$ such that $v$ stores only one level of information and $v$ is the nearest sink among any cluster head $u \in C_{i}$, then $v$ is parent cluster head of $C_{i}$. Otherwise, redirect the task to a cluster head $w$, where $w$ is the cluster heads closet to sink among all 1-hop neighbors of $C_{i}$. The sink is assigned to be parent in highest level

The proposed scheme can be illustrated as the following example. An illustrative example shows in Figure 2. Every square represents a cluster head. The square marked $k$ is the cluster head stores the information of level 0 and level $k$. Sink is in the bottom of the network. Arrows represent the data flow from low level cluster heads to its parent. Consider the circled level-2 cluster head, the circle level-2 cluster head in the marked region is the cluster head nearest to sink among circled level-2 cluster heads, but it already stores two levels of information and then redirects the task to its right neighbor. Its right neighbor stores only level 0 information and then become the parent of circled level-2 cluster heads.

To prove our scheme is storage load-balanced, we evaluate the variance of storage cost. Suppose the height of the hierarchy is $k$, the expected value of the storage cost for each cluster head is $E_{H T M}=\frac{4-4^{-k}}{3}$. Then we can evaluate the variance of the storage cost as:

$$
\begin{aligned}
V_{H T M} & =\frac{\left(2-E_{H T M}\right)^{2}\left(\frac{4^{k}-1}{3}\right)+\left(1-E_{H T M}\right)^{2}\left(\frac{2 \times 4^{k}+1}{3}\right)}{4^{k}} \\
& =\frac{2}{9}-\frac{4^{-k}}{9}-\frac{4^{-2 k}}{9}=\Theta(1)
\end{aligned}
$$

Similarly, we can evaluate the storage cost for each cluster head using conventional clustering hierarchy architectures to be $\Theta\left(k^{2}\right)$. It can be seen that $V_{H T M}$ grows in the constant rate and is independent to the height. Therefore we can conclude that the proposed scheme can satisfy the storage load-balance and thus ensure the quality of moving patterns for each cluster head.

\subsection{Storage Replacement Strategy}

Through the proposed storage scheme can ensure that a level $i(i \geq 1)$ cluster head only has to maintain two levels of information, with time passing by, the storage of each cluster head will run out. Therefore, we must to discard less

\footnotetext{
${ }^{1}$ That is, every parent cluster head in hierarhcy has four children.
}

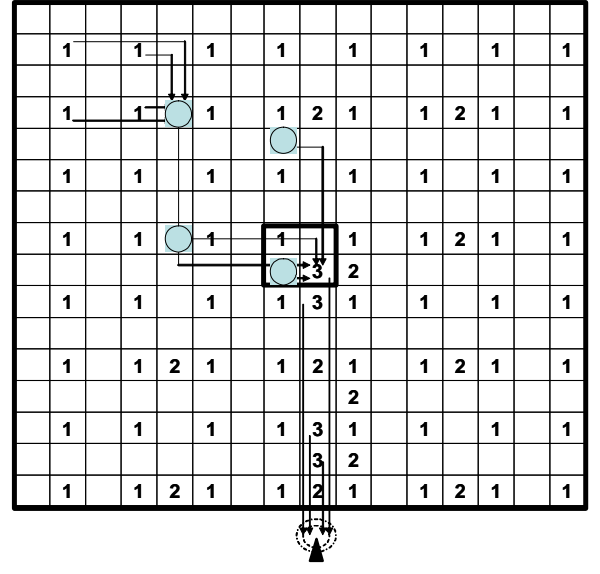

Figure 2. Storage load-balanced scheme for
HTM.

informative patterns to store more informative ones. Hence, the storage replacement strategy is necessary for dealing with this situation. When the memory space of a cluster head is full and the count of one symbol in the table maintained by an emission tree node is larger than given threshold min_sup, we have to decide to prune other nodes so that the newborn node can be inserted into emission trees. We specify a threshold $\epsilon$ and if the prediction hit rate of the tree to which the newborn node belongs is already larger than $\epsilon$, we can ignore the insertion since this emission tree already has higher prediction rates. Otherwise, we must select an appropriate node to be pruned from other trees. The pruning mechanism consists two steps:

1) Select the tree to be pruned. Each object usually has its own moving behavior. An object may stay in some regions more frequently than other regions. Hence, the reporting rates of objects in each cluster head will be different. For tree selection, each tree maintains a counter. The counter increases one when a tree is updated and minuses one every $\mathrm{T}$ periods. Obviously, a tree with a lower counter value means that it is not often used than other trees. Once a newborn generated, we only select the same level tree to the newborn node to prune. To guarantee that the accuracy of each tree is acceptable, we specify a threshold $\xi$. Suppose that the new node will be inserted into a level $i$ emission tree. Among other level $i$ trees with their prediction hit rates $\geq \xi$, the tree with the minimal counter value will be selected. Consider an example in Figure 3, where the size of a tree stands for the access counter value of the tree. Let $\epsilon=0.8$ and $\xi=0.6$. Since the hit rate of tree $T_{1}<0.8$, we decide to insert the new node into $T_{1}$ and prune one node in $T_{2} \sim T_{5} . T_{2}$ will not be selected since its hit rate $<0.6$ and $T_{4}$ is next selected. Since the hit rate of $T_{4}>0.6, T_{4}$ is then 
selected to be pruned.

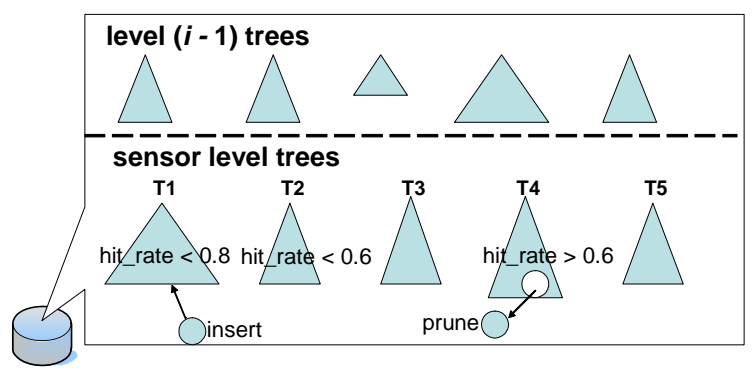

Figure 3. An illstrative diagram of pruning node in a level i cluster head when its memory space is full and the new node is decided to be inserted.

2) Select the node which will be replaced by the new node. Once the tree is selected, we must prune the node such that there will be less impact to the selected emission tree. Let LNode is the set of all leaf nodes which no other node is derived from them. For example, assume that node $C D$ and $C D A$ are leaf nodes, node CDA belongs to LNode. For the node selection, each tree maintains the profits for LNodes. To derive the profit function for a node, two factors, the probability and the mature degree, of a node is used to evaluate the importance of a node. The mature degree of a node $x$ is defined as $M D(x)=\frac{N}{\beta}$, where $\mathrm{N}$ is the number of times that the $L_{\infty}$ distance of the probability distribution of node $x$. Obviously, the higher the two factors are, the more important the node is. Therefore, given $c$ as a real constant used as the base, we define the profit function for a node as:

$$
\operatorname{Profit}(x)=P(x) \times(M D(x)+c)
$$

The node with the minimal profit value in LNode will be chose to be pruned. Moreover, to reduce the cost of maintaining LNode, if a node becomes mature, we won't continue to update the probability entries in the table of the node. For example, suppose node DCAE is a node in LNode and node $D C$ and node DCA are immature. We don't have to recalculate $P(D C A E)$ until one of node $D C$ and node $D C A$ becomes mature.

With a storage replacement strategy, there will not be a specific tree which is always selected to be pruned. Since if the prediction hit rate of a tree becomes $<\varepsilon$, the tree will not be selected anymore. Furthermore, even if the hit rate of a tree becomes lower due to the node pruning, it still has chances that the nodes can be inserted back.

\section{Performance Study}

In this section, experimental results of our performance study (based on simulation) are presented. The simulation model is described in Section 4.1. The comparison of our scheme with PES scheme [5] is conducted in Section 4.2. Finally, the sensitivity analysis of our proposed storage replacement strategy is described in Section 4.3.

\subsection{Simulation Model}

In the experiment, we consider a three-level heterogeneous tracking model, where 9 low-end sensors are deployed in each level-0 cluster. Hence, there are 16 level0 cluster head, 4 level- 1 cluster heads, one level-2 cluster head, and the number of low-end sensors is 144 . To simulate the object movements, we generate VMM model trees for each object in each cluster head. In addition, the city mobility model [3] is used to simulate object movements with locality. With the model, each object has a probability $p_{1}$ to determine whether it should leave its current level-1 cluster, and a probability $1-p_{1}$ to stay. In the former case, it will choose a level-1 cluster as the next position according to its VMM model tree in the level-2 CH (It may stay in the current level-1 cluster). In the latter case, it has a probability $p_{0}$ to determine whether it should leave its current level-0 cluster, and a probability $1-p_{0}$ to stay. Similarly, in the former case, it will choose a level-0 cluster as the next position according to its VMM model tree in the parent. In the latter case, it will stay in its current level-0 cluster. In all cases above, the VMM model looking up procedure is repeated until the object has decided to move to which low-end sensor monitored region. The probability $p_{i}$ is determined by an exponential probability $p_{i}=e^{-C \cdot 2^{i+1}}$, where $C$ is a positive constant. A higher value of $C$ means higher locality. The value of $\delta$ used to justify whether the cluster head shall be in the prediction phase is set to 0.5 and the probability threshold for the number of senors in the prediction phase (i.e., $\gamma$ ) is set to 0.2 .

\subsection{Experiments of PES and HTM}

In this experiment, we compare our object tracking models with load-balance storage scheme denoted as HTM with PES scheme [5]. In order to show the proposed loadbalance storage scheme, we also implement our tracking model without load-balance storage scheme, expressed as HTM w/o). To conduct the experiments of PES scheme in [5], each object will change its speed and direction every 5 seconds and employ the INSTANT heuristic for prediction. The sampling and reporting frequency are once per second. Once the prediction is not correct, the recovery procedure 


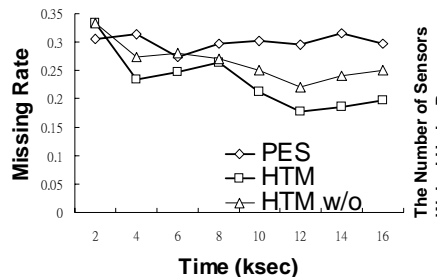

(a)

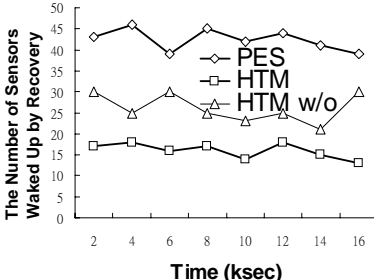

(b)
Figure 4. (a) The average missing rates between PES, HTM and HTM w/o (b) The average number of nodes participated in the recovery procedure.

will be performed by waking up sensor nodes. The missing rate of two scheme is shown in Figure 4(a).

The number of sensor nodes waken up under PES and HTM is shown in Figure 4(b). By utilizing object moving patterns, HTM is able to accurately predict the movements of objects and then the number of executing the recovery procedure is small. Note that the impact of storage balance to moving patterns is shown. In HTM w/o, cluster heads that store too many levels of information will reduce the accuracy of prediction. Furthermore, due to the very hierarchical nature of HTM, once the recovery procedure is performed, only a bound number of sensors activate for tracking objects. Thus we can conclude that load-balance storage scheme improves the accuracy of prediction.

\subsection{Sensitivity Analysis of Storage Replacement Strategy}

To conduct the experiments of various $\beta$ for emission tree node maturity verification, the value of $\alpha$ is set to 0.01 . Figure 5 shows the predication rates with the value of $\beta$ varied. As can be seen in Figure 5, the value of $\beta$ should not set too small or too large. If the emission tree training just gets starting, it is possible that the probability difference is very small when the cluster head receives the same record for a few times. Thus, with a smaller value of $\beta$, tree nodes do not collect sufficient moving information for prediction. Once the value of $\beta$ is too large, tree nodes are hard to become mature nodes. Even though tree nodes collect enough moving information, these tree nodes are not able to use for prediction. Clearly, the selection of $\beta$ will be dependent upon the moving behavior of objects and can be determined empirically.

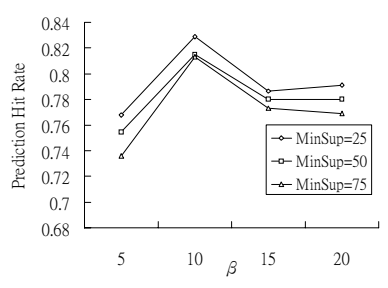

\section{Figure 5. The impact of $\beta$ for emission tree node maturity verification.}

\section{Conclusions}

In this paper, we proposed a heterogeneous tracking model, called HTM, to efficiently mine object moving patterns and track objects. Since HTM relies on moving patterns to predict, storage management is an important issue. In this paper, we proposed a load-balance storage scheme for HTM that satisfies storage load-balanced and thus ensure the quality of moving patterns in each cluster head. Furthermore, when a storage of a cluster head is full, we also developed a storage replacement strategy. Simulation results show that HTM with our proposed storage management can achieve the best performance to improve the accuracy and thus saves energy for tracking objects.

\section{References}

[1] M.-S. Chen, J. Han, and P. S.Yu. Data Mining: An Overview from Database Perspective. IEEE Transactions on Knowledge and Data Engineering, 8(6):866883, December 1996.

[2] M. Y. et al. Exploiting heterogeneity in sensor networks. In Proceedings of 24th Annual Joint Conference of the IEEE Computer and Communications Societies (INFOCOM 2005), 2005.

[3] C.-Y. Lin and Y.-C. Tseng. Structures for in-network moving object tracking in wireless sensor networks. In BROADNETS, pages 718-727. IEEE Computer Society, 2004.

[4] W.-C. Peng and M.-S. Chen. Developing Data Allocation Schemes by Incremental Mining of User Moving Patterns in a Mobile Computing System. 15(6), 2003.

[5] Y. Xu, J. Winter, and W. C. Lee. Prediction-based Strategies for Energy Saving in Object Tracking Sensor Networks. In Proceedings of the 2004 IEEE International Conference on Mobile Data Management(MDM'04), 2004

[6] J. Yang and W. Wang. Agile: A general approach to detect transitions in evolving data streams. In Proceedings of ICDM, pages 559-562. IEEE Computer Society, 2004. 